\title{
TMEM16C cuts pain no SLACK
}

\author{
Vinicius M Gadotti \& Gerald W Zamponi \\ TMEM16C has an unexpected role in regulating the activity and cell surface expression of sodium-activated \\ potassium (SLACK) channels. By enhancing SLACK currents, TMEM16C indirectly inhibits pain signaling.
}

Neuronal activity in pain-sensing neurons depends on the function of a wide range of ion channels, including sodium, calcium, potassium and chloride channels. There is an intricate balance between excitatory and inhibitory conductances that maintains appropriate levels of excitability in afferent nociceptive fibers. Perturbations in ion channel trafficking and/or function can perturb this equilibrium, altering neuronal firing and giving rise to pain hypersensitivity. A recent study identified the calcium-activated chloride channel TMEM16A (also known as ANO1) as an important regulator of dorsal root ganglion (DRG) neuron excitability and heat sensitivity ${ }^{1}$. This channel belongs to the family of anoctamins, a group of ten homologous membrane-spanning proteins that includes several chloride channels and some members whose physiological roles and functions are not understood ${ }^{2-4}$. In this issue of Nature Neuroscience, Huang et al..$^{5}$ identify an enigmatic member of this family, TMEM16C (ANO3), as regulating the electrophysiological properties of nociceptive neurons in a manner strikingly different from that reported for TMEM16A. Unlike the putative direct ionotropic function of the latter, TMEM16C acts indirectly by regulating sodium activated potassium channel $\left(\mathrm{K}_{\mathrm{Na}}\right)$ trafficking and function, resulting in the regulation of pain signaling.

The authors first examined the cellular distribution of TMEM16C channels by using a newly generated antibody and found robust expression in small nociceptive neurons. They confirmed their results using reverse transcription-PCR analysis. Next, they generated a TMEM16C knockout rat to determine whether the high expression of TMEM16C in nociceptive neurons may be linked to pain signaling. Indeed, the authors found that the knockout rat showed a reduced threshold for thermal and mechanical pain. Consistent with these findings, the threshold for action potential firing was reduced in DRG neurons from TMEM16C knockout rats. This is diametrically opposed to what has been described for the ANO1 channel ${ }^{1}$, suggesting a

Vinicius M. Gadotti and Gerald W. Zamponi are in the Department of Physiology and Pharmacology, Hotchkiss Brain Institute, University of Calgary, Calgary, Alberta, Canada.

e-mail: zamponi@ucalgary.ca mechanism of TMEM16C action that is different from a putative chloride channel activity.

To determine how TMEM16C alters DRG neuron function, the authors conducted electrophysiological analyses in both neurons and heterologous expression systems. As perhaps expected, the authors did not detect any alterations in chloride conductances following either overexpression or depletion of TMEM16C. During their analysis, however, they stumbled across an intriguing alteration in whole-cell potassium conductance that is mediated by the $\mathrm{K}_{\mathrm{Na}}$ channel SLACK. In TMEM16C knockout rats, SLACK currents and membrane protein levels were reduced. Conversely, coexpression of TMEM16C with SLACK in HEK293 cells yielded an increase in SLACK currents that could be attributed to an increase in both the number of SLACK channels present on the membrane and the probability of each of them being open, the latter being mediated by an enhanced sensitivity of the channel to the agonist sodium. Consistent with a direct regulation of SLACK function by TMEM16C, these two proteins appeared to exist as a biochemical complex.
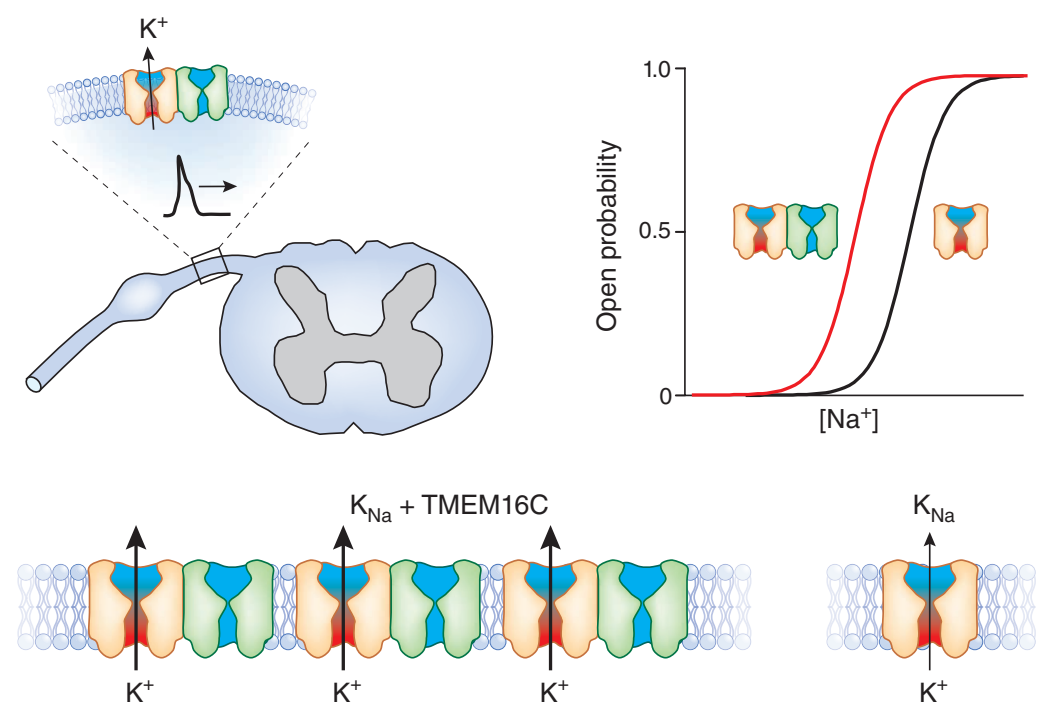

Figure 1 Regulation of SLACK channels by TMEM16C. Top left, spinal cord slice with an attached DRG. SLACK channels $\left(\mathrm{K}_{\mathrm{Na}}\right)$ and TMEM16C channels are expressed in DRG neurons, where they shape action potentials. Top right, co-assembly of SLACK with TMEM16C leads to a leftward shift in the sodium dose dependence of SLACK channel activation (red line) relative to that of SLACK alone (black), yielding higher open probability at lower sodium concentrations. Bottom, co-assembly of SLACK with TMEM16C (left) results in more SLACK channels in the plasma membrane than in the absence of TMEM16C (right). Together with the increase in open probability, this results in greater whole-cell potassium currents. 
The intriguing results of Huang et al..$^{5}$ underscore the importance of proper ion channel expression and function in limiting the basal excitability of afferent pain fibers, but they also raise questions. First, is TMEM16C downregulated under conditions of chronic pain and, if so, is this partially responsible for the associated increase in neuronal excitability? Second, given that TMEM16C and SLACK are also present in tissues, such as the $\mathrm{CNS}^{7,8}$ and heart, might a similar TMEM16CSLACK-mediated regulation of cellular excitability occur more widely? Third, could this mechanism contribute to other pathophysiologies associated with altered SLACK function, such as epilepsy ${ }^{9}$ ? Fourth, does knocking down SLACK in mice reduce pain thresholds? At a mechanistic level, it remains unclear whether the interaction with TMEM16C results in an increased affinity of the SLACK channels for sodium ions or simply a greater sensitivity of the activation mechanism to this ionic agonist. Moreover, it remains to be determined whether the TMEM16C-mediated increase in membrane expression of SLACK is a result of effects on gene transcription, enhanced endoplasmic reticulum export or a stabilization of the protein complex at the plasma membrane. This last mechanism may be supported by observations that the internalization of SLACK channels is under dynamic secondmessenger control ${ }^{6}$. Despite these open questions, the work of Huang et al..$^{5}$ is exciting and sets the stage for further exploration.

The functions of the TMEM16 (ANO) family of transmembrane signaling molecules are only incompletely understood. TMEM16A (ANO1), TMEM16B (ANO2) and TMEM16F (ANO6) have been described as small-conductance calcium-activated chloride channels ${ }^{10-12}$ that help regulate chloride homeostasis. In contrast, most other members of this family do not appear to support chloride channel activity, despite a high degree of homology with those that $\mathrm{do}^{4}$. These chloride-impermeant members of the TMEM16 family may well mediate functions such as those described by Huang et al. ${ }^{5}$. Perhaps more intriguing is a scenario in which ANO proteins that are known to support a chloride conductance may also act in a manner that is reminiscent of TMEM16C; that is, by modulating other ion channels in a non-ionotropic manner. Irrespective of this possibility, the fact that TMEM16C acts as a modulator of $\mathrm{K}_{\mathrm{Na}}$ channel function indicates a previously unrecognized role of the ANO family in regulating ion channel activity that demands a fresh look at this class of transmembrane molecules.

\section{COMPETING FINANCIAL INTERESTS}

The authors declare no competing financial interests.

1. Cho, H. et al. Nat. Neurosci. 15, 1015-1021 (2012). 2. Tian, Y., Schreiber, R. \& Kunzelmann, K. J. Cell Sci. 125, 4991-4998 (2012).

3. Duran, C., Qu, Z., Osunkoya, A.O., Cui, Y. \& Hartzell, H.C. Am. J. Physiol. Cell Physiol. 302, C482-C493 (2012).

4. Milenkovic, V.M., Brockmann, M., Stöhr, H., Weber, B.H. \& Strauss, O. BMC Evol. Biol. 10, 319 (2010).

5. Huang, F. et al. Nat. Neurosci. 16, 1284-1290 (2013)

6. Nuwer, M.O., Picchione, K.E. \& Bhattacharjee, A. J. Neurosci. 30, 14165-14172 (2010).

7. Santi, C.M. et al. J. Neurosci. 26, 5059-5068 (2006).

8. Charlesworth, G. et al. Am. J. Hum. Genet. 91, 1041-1050 (2012).

9. Heron, S.E. et al. Nat. Genet. 44, 1188-1190 (2012).

10. Yang, Y.D. et al. Nature 455, 1210-1215 (2008).

11. Caputo, A. et al. Science 322, 590-594 (2008).

12. Yang, H. et al. Cell 151, 111-122 (2012).

\title{
A finely tuned cortical amplifier
}

\author{
Yunyun Han \& Thomas Mrsic-Flogel
}

\section{Three studies in visual and auditory cortex show that intracortical excitatory inputs amplify incoming sensory signals, as their sensory tuning is closely matched to that arriving from the sensory thalamus.}

One of the longest-standing debates in neuroscience centers on how preference of individual neurons for sensory stimuli arise in the neocortex. In dedicated areas of sensory cortex, neurons are specialized to detect particular sensory features, such as the orientation and motion direction of edges in images, or the spectral content of sounds. Remarkably, anatomical studies have shown that a neuron in the cortex receives only a tiny fraction of its excitatory synapses from thalamic regions conveying information from the sensory organs ${ }^{1}$, whereas the rest are largely provided by other cortical neurons embedded in a richly connected circuit. Our understanding of the relative contribution of thalamic and intracortical inputs in shaping neuronal response properties is far from complete. In this issue, three studies shed light, quite literally, on this question.

The establishment of sensory feature selectivity is perhaps best understood in primary

Yunyun Han and Thomas Mrsic-Flogel are at the Biozentrum, University of Basel, Basel, Switzerland. e-mail: thomas.mrsic-flogel@unibas.ch visual cortex (V1). Classically, the selectivity for oriented bars or edges has been explained using Hubel and Wiesel's 1962 model, whereby 'simple' cells in cortical layer 4 first become selective for stimulus orientation or direction via the convergence of $\mathrm{ON}$-center and OFF-center thalamic inputs whose receptive fields are offset in visual space, but are themselves not orientation or direction selective. In cat V1, each cortical column receives input from a limited subset of neurons in the lateral geniculate nucleus (LGN) of the thalamus, and the angle of apposition of ON and OFF domains determines the orientation preference of neurons in that column ${ }^{2,3}$. To isolate the contribution of thalamocortical input to cortical feature selectivity, previous studies have measured synaptic drive while silencing cortical activity by means of cooling or electric shocks ${ }^{4,5}$. These studies found that input from the thalamus is sufficiently tuned to establish the orientation selectivity of thalamorecipient cortical neurons.

What, then, is the contribution of intracortical connections to sensory responses of cortical neurons? Computational models informed by anatomy suggest that one role of intracortical connections is to amplify thalamocortical input (for example, ref. 6). This will depend on the strength and specificity by which cortical neurons connect to each other. We know that cortical connections are not randomly organized, as excitatory neurons with similar visual responses connect to each other preferentially (but not exclusively) in layer $2 / 3$ of mouse and cat V1 (refs. 7,8). This suggests that neurons form partially overlapping subnetworks within which recurrent excitation may boost the responses more to preferred than to non-preferred stimuli.

The three new studies have now directly estimated the relative influence of feedforward and intracortical excitation to sensory evoked responses in layer 4 of mouse visual and auditory cortex ${ }^{9-11}$. These studies used in vivo voltage-clamp recordings in single neurons in combination with rapid and reversible silencing of cortical excitatory activity, which enabled them to isolate putative thalamocortical and intracortical components contributing 